\title{
THE USE OF INSULIN IN THE TREATMENT OF CORNEAL ULCERS*
}

\author{
BY
}

\section{T. R. AyNSLEY}

BOURNEMOUTH

THE treatment of ulcers of the cornea by the usual methods of chemical or thermal cauterization, plus the use of the sulphonamide preparations (and more recently penicillin) is as a rule satisfactory and adequate, but there are still occasional cases which are troublesome and I should like to report a few in which I have thought the use of insulin beneficial in promoting more rapid healing than appeared otherwise likely.

CASE 1.-In 1937 I was treating a man with right facial paralysis of old standing who had ectropion and associated chronic conjunctivitis. He had developed a large corneal ulcer and was admitted to hospital.

'The ulcer was carbolized and various drops were used for nine days without improvement. It was then I suggested using insulin as drops, and two or three days later when I saw him, though the site of the ulcer looked white and " soggy," much to my surprise it did not stain with fluorescein. There seemed to have been an excessive proliferation of epithelium. He made an uninterrupted recovery.

The sequel to this case is that a year later he came under the care of another ophthalmic surgeon who asked me to see him after a fortnight of unavailing treatment. I recognised the man, who was then given insulin drops to use as an out-patient. The ulcer was quite healed when I saw him the following week: I have seen him again this year and there has been no recurrence.

Since that time I have used insulin both as drops and by injection in any case which did not respond to the usual methods of treatment. Latterly I have confined the insulin treatment to the injection of five units daily, which, if used on out-patient cases, is accompanied by the oral exhibition of glucose.

CASE 2.-A Service case with burns of face and severe general injuries.

The left eye was blind and the right eye showed a large exposure ulcer occupying the lower third of the cornea up to the margin of the pupil. Complete apposition of the lids was impossible owing to burns and sepsis.

Treatment with albucid drops, and later mercurochrome, and saline irrigations was carried out for two or three weeks without improvement. The patient was then put on injections of insulin

* Received for publication, February 10, 1945. 
and showed no change for four days. I examined him while he was having à general anaesthetic one morning and his ulcer seemed exactly the same size, and stained throughout with fluorescein. Two days later it had completely healed except for a hair-line of staining round the periphery.

CASE 3.-A child, aged two, with severe Pink Disease.

Most of the child's hair had come out, the chest was full of râles, and both first and second teeth were being shed, the latter due to necrosis of the jaw. The right eye showed a superficial ulcer covering about five-eighths of the cornea.

Three units of insulin were given daily and four days later the ulcer was healed. In view of the very poor general condition of the child, I was inclined to think that the eye might be lost. No carbolization was done.

CASE 4.-I here include notes of a case which Mr. Basil Graves had under his care, which he has kindly made available.

" A patient had had chronic superficial ulceration of the right cornea in 1915 and in 1925 . An excavating ulcer occurred in 1944, not far from the centre of the cornea. There was very little ciliary injection.

" The eye persisted in this state for months, and in spite of the trial of various customary local and general treatments, there was no sign of improvement as adjudged by slit-lamp examination. Insulin was recommended, but at first was not used because the patient's doctor was disinclined to give it. After another six weeks, as no sign of improvement was evident, injections of insulin were given. The ulcer started to heal and within about two or three weeks the whole excavation was filled in with epithelium to the level of the normal corneal contour. This was four months ago : the eye has remained quiet and normal since."

CASE 5. A man with severe herpes frontalis. The right eye was blind from an old injury. The left eye showed an ulcer extending over most of the cornea except for a small area above the pupil up and in. Some blood-stained hypopyon was present and the iris vessels injected. The tension was raised and there was chronic conjunctivitis.

The lids were painted with 1 per cent. silver nitrate, and he was treated with albucid drops and albucid by mouth. Five units of insulin were given daily. There was no improvement for two days, but on the fourth day, when I asked a colleague to see him with a view to getting an opinion as to whether a tarsorrhaphy should be done, we found the cornea healed. No carbolizing had been done. A slight relapse took place a few weeks later but healing again occurred and has been maintained.

I have quoted only a few cases, more or less as samples. Insulin may possibly have been used before in ophthalmology, but I have no references available. Since the war there.has been 
at least one mention in the British Medical Journal of its use in promoting more rapid healing of extensive wounds of the limbs, and my house surgeon has recently called my attention to a very brief reference to its use in indolent ulcers. (Pye's Surgical Handicraft.)

There would appear to be some scope for further investigation of its action in hospitals with more abundant material and facilities for controlled experiments.

Three possibilities suggest themselves to me. Either insulin improves the patient's nutrition, or removes some factor necessary for germ-metabolism, or it increases the rate of epithelial proliferation. If so it may be worth a more extensive trial,in war surgery as well as ophthalmology.

\title{
TWO RARE CASES OF HOMOPLASTIC SURGERY OF THE EYELIDS*
}

\author{
BY \\ N. I. SHIMKIN \\ HAIFA, PALESTINE
}

Two successful cases of homoplastic surgery of the eyelids are described, both having unusual clinical features.

The first case.-Post-trachomatous trichiasis in a haemophilic youth was treated by a graft from the buccal mucous membrane of his father. In the ophthalmic literature which I have consulted, I have not been able to trace a single similar case.

The second case.-Four lid ichthyosis ectropion in a baby of 13 months suffering from a congenital generalised ichthyosis was treated with whole skin grafts from the forearm of his mother. Four lid ectropion in congenital ichthyosis has been seldom described but Elschnig ${ }^{2}$ reports a successfully treated case in 1912 , published in 1923.

\section{Case I}

The patient, a Moslem Arab, aged 22 years, of good general condition, consulted me on January 28, 1944. On examination :Right eye : Trachoma III. Pannus levis, central nebula, vision -6/60. Left eye : Blepharospasm, photophobia, trachoma III, trichiasis gravis, pannus crassus ulcerosus. Vision : hand movement before the eye.

The patient was advised to be operated on for trichiasis. He consented and asked for the operation to be performed immediately, as he was afraid of losing the sight of the left eye altogether. 